[10] R. M. Washburn et C. H. Jones. Vermont Agric. Exp. Sta. Bull., $1916,195$.

[11] A. C. Willatd et K. Blunt. Journal Biol. Chem., 1927, 75, 251.

\title{
UNE MÉTHODE SINACIDE \\ DE DOSAGE DE LA MIATIÈRE GRASSE DU LAIT UTILISANT LE MATÉRIEL GERBER (1)
}

\author{
par \\ J. C. GODFRAIN \\ Professeur à l'Ecole Nationale Vétérinaire de Toulouse
}

Le dosage de la matière grasse du lait, demandé de plus en plus fréquemment aux laboratoires, depuis le paiement du lait aux producteurs en fonction du taux de cette matière grasse, entraîne, pour ces laboratoires, la nécessité d'un personnel ne réalisant que ce dosage. Ce personnel, pour des raisons financières très compréhensibles, ne peut pas être hautement qualifié, et il importe de lui confier une tâche simple et surtout dénuée de tout danger.

Cependant, de très nombreuses techniques de dosage proposées ne répondent pas à ces critères et la méthode Gerber, issue du procédé américain Babcok, utilisée dans l'ensemble des laboratoires français, si elle est rapide, n'est pas sans inconvénients. Le matériel nécessaire est relativement coûteux, mais surtout la manipulation d'acide sulfurique concentré a déjà donné lieu à des accidents dus, pour une bonne part, à la fragilité des butyromètres, au mode de bouchage de ceux-ci ainsi qu'au nombre d'opérations nécessaires. $\mathrm{Si}$ on ajoute que les appareils mesureurs automatiques de l'acide sulfurique sont loin d'être étanches (pertes d'acide sur les tables de travail; hydratation de l'acide dans le réservoir), on s'aperçoit que la mise au point d'une nouvelle méthode de dosage est souhaitable.

Le problème que nous avons cherché à résoudre était done le suivant : réaliser le dosage de la matière grasse à l'aide du matériel dont les laboratoires disposent couramment, sans utiliser les liqueurs caustiques.

Dans la résolution de ce problème, nous avons été guidé par les travaux de Schain et de Gershenfeld notamment. Les techniques suggérées par ces auteurs sont basées sur l'utilisation des détergents pour briser la couche protectrice des globules gras et permettre la formation d'une couche continue. Les détergents utilisés sont de deux types : l'un non ionisé, l'autre anionique.

(1) Revue de Médecine Vétérinaire, 1955, 106, 370. 
Pour faciliter la lecture, diverses matières colorantes non hydrosolubles et liposolubles sont utilisées.

A partir de ces éléments nous nous sommes efforcé de réaliser une méthode analogue aux précédentes, mais utilisant le matériel Gerber et les réactifs que nous étions susceptibles de trouver commodément en France.

A la suite des nombreux essais que nous avons faits, nous nous sommes arrêtés aux réactifs et à la technique ci-dessous :

\section{Réactifs}

10 Solution colorante : elle" est préparée par saturation en Ecarlate R (R.A.Ĺ.) d'alcool amylique;

$2^{\circ}$ Solution détergente : elle est constituée à l'aide : a) d'un détergent non ionique, le Poly-éthoxy-éther de monopalmitate de sorbitan $(1) ; b$ ) d'un détergent anionique de sel de sodium de l'acide dioctylphosphorique (2).

Ces deux détergents sont mélangés à raison de $20 \mathrm{~cm}^{3}$ de a) pour $100 \mathrm{~cm}^{3}$ de b). Le mélange est coloré par $2 \mathrm{~cm}^{3}$ de la solution Ecarlate R.

\section{Technique}

$11 \mathrm{em}^{3}$ de lait sont placés dans le butyromètre ; on ajoute, sans précaution spéciale, $8 \mathrm{~cm}^{3}$ du mélange des détergents et $2 \mathrm{~cm}^{3}$ d'eau distillée (chaude si possible).

On bouche le butyromètre on le secoue fortement de telle sorte que les liquides soient totalement et intimement mélangés et on le place au bain-marie à $100^{\circ}$ pendant six minutes.

Le butyromètre est ensuite centrifugé à 1.500-2.000 tours, pendant cinq minutes, puis placé au bain-marie à $70^{\circ}$, pendant einq minutes.

La couche graisseuse apparaît colorée en rose groseille nettement tranchée sur le reste du liquide, qui est blanchâtre. La lecture se réalise done sans aucune difficulté.

Nous avons essayé de remplacer la centrifugation par le chauffage prolongé, mais nous n'avons pas obtenu de résultat valable avant vingt-quatre heures, délai trop long pour rendre le procédé utilisable.

Les mesures que nous avons faites suivant cette technique sont tout à fait comparables à celles réalisées à l'aide de la méthode Gerber, à condition de respecter les temps de chauffage et de centrifugation.

Nous envisageons l'extension de notre procédé de dosage de la matière grasse à d'autres produits laitiers.

(1) Tween 40 Atlas Powder Co, à Wilmington (U.S.A.).

(2) Tergitol P, 28 de Union Carbide International Co, à New York. 


\title{
BIBLIOGRAPHIE
}

[1] P. Schain. Science, 1949, 110, 121.

[2] L. Gershenfeld and B. I. Ucko. Milk and food Technology, 1950, 13, 175.

[3] J. Pien. "Vues actuelles sur le dosage de la matière grasse du lait ", dáns Mises au point de chimie analytique pure et appliquée et d'analyses bromatologiques, publiées sous la direction de J. A. Gadtier, $3^{\text {e }}$ série, 1 volume de 192 pages, Masson éditeur, 120, Boulevard SaintGermain, Paris $\left(6^{\mathrm{e}}\right) .1955$.

[4] F. Aftalion. Les polyoxyéthylèneglycols en cosmétologie. La Parfumerie moderne, 1953, no 34 , juillet-août.

[5] J. P. Sisley. Index des huiles sulfonées et détergents modernes, p. 535. Paris, 1949.

\section{COMPOSITION EN VITAMINE A \\ ET EN CAROTËNE DE BEURRES DE CONSOMIMATION DE L'ÉTAT DE SAO PAULO (BRÉSIL)}

\author{
par
Professeur P. MUCCIOLO, O. BARBUTO, M. M. CAMPOS
Directeur
Technologiste
Chimiste

Laboratoire du "Département de l'Industrie, inspection et conservation des produits alimentaires d'origine animale», de la Faculté de Médecine Vétérinaire de l'Université de Sao Paulo (Brésil)

Notre but, en entreprenant ce travail, était de connaître la composition en vitamine $\mathrm{A}$ et en carotène des beurres fabriqués dans l'Etat de Sao Paulo. Des enquêtes de cette catégorie ont déjà été réalisées dans de nombreux pays, et il n'est pas nécessaire d'insister sur leur importance pratique. Dans l'Etat de Sao Paulo, nous n'avons trouvé, comme étude semblable, qu'une seule étude, de Cecy Mello Texeira [1] présentant les moyennes de 61 analyses effectuées sur des beurres recueillis au marché de la ville de Sao Paulo.

Désirant étendre cette enquête, et surtout observer les variations de composition dues aux époques différentes de l'année, nous avons eu de grosses difficultés relativement à l'obtention des échantillons en temps voulu, ce qui nous a privé des éléments nécessaires pour pouvoir en toute sécurité présenter l'analyse statistique de nos résultats. Dans les conditions où nous avons opéré, nous ne pouvons prétendre que nos résultats peuvent être pris comme règle générale, malgré la quantité appréciable de matériel analysé, provenant de presque tous les centres importants de production de beurre de l'Etat de Sao Paulo, dont quelques-uns de l'Etat du Minas Gérais qui fournissent également la ville de Sao Paulo. 